\title{
Assessment of Mothers' Knowledge about Breast Feeding, Bottle Feeding and Weaning of their Infants Attending Maternal Child Health Centers at Minia City
}

\author{
El shaimaa G. Hassan, Salah M. Salah, Asmaa A. Mohamoud, \& Eman S. Masoed. \\ Pediatric Nursing, Faculty of Nursing, Minia University. \\ Pediatrics, Faculty of Medicine, Minia University. \\ Pediatric Nursing, Faculty of Nursing, Assuit University.
}

\begin{abstract}
Aim of The study to assess the mothers' knowledge about breast Feeding, bottle Feeding and weaning of their Infants. This study was carried out in all $\mathrm{MCH}$ centers at Minia city. Descriptive research design was used to meet the aim of study. A convenient sample included 1000 mothers who were attending MCH centers. A structured interview sheet was developed by the researcher based on review of relevant literature. It included 2 parts: Part I; included sociodemographic characteristics of the mother and child, Part II; included items related to mothers' knowledge about breast feeding, bottle feeding, and weaning. There were highly statistical significant differences between total knowledge of mother about breast feeding and all her socio-demographic characteristics. There was a positive relationship between maternal education and increased knowledge about infant feeding. Conclusion of this study illustrated that there is a relationship between knowledge of mother about breast feeding, bottle feeding ,and weaning and her socio-demographic characteristics and that there is a relationship between the education of mother with her knowledge about pattern of breast feeding, bottle feeding, and weaning and that there is a relationship between the occupation of mother and knowledge about exclusive breast feeding, duration of exclusive breast feeding. The study recommended that there is a dire need to arrange for health education program sessions for mothers with main emphasis on importance of colostrum's feeding, exclusive breast feeding \& suitable age of starting and complete weaning.
\end{abstract}

Key words: Nutrition, Infants, Breastfeeding, Bottle feeding \&Weaning.

\section{Introduction}

Infancy is defined as the period from birth to 12 months of age (Ricci and Kyle, 2009). Babies under 1 year get most of their vitamins and minerals from breast milk or formula. When solid foods are introduced they are to supplement, not replace milk. Only gradually should solid foods become major sources of nutrients (Food and Nutrition Board, National Academy of Sciences-National Research Council, 2003).

Growth and developmental changes in the first year of life are numerous and dramatic. Adequate growth and development are indicative of health in infant or young child. The newborn and infant's organ systems undergo significant changes as the infant grows. Systems that undergo significant change include the neurologic system, the cardiovascular system, the gastrointestinal (digestive) system, the renal system, the hematopoietic system, and the immunologic system (Kyle, 2008).

Adequate nutrition is essential for growth and development. Breast feeding and bottle-feeding of infant formula are both acceptable means of nutrition in the newborn and infant. Breast milk or formula supplies all of the infant's daily nutritional requirements until 4 to 6 months of age, at which time solid foods may be introduced (Ricci and Kyle, 2009).

Inadequate nutrition can disrupt cognition and intellectual functioning. In a review of the published literature, (Martorell, 1997) concluded that poor nutrition during early childhood, especially in developing country settings, can have varied effects on intellectual functioning. Our knowledge of the mechanisms by which malnutrition during these years disrupts these functional outcomes, however, is still evolving (Levitsky and Strupp, 1995).

The causes of malnutrition are numerous. These causes are intertwined with each other and are hierarchically related. The most immediate (or proximate) determinants of malnutrition are poor diet and illness. Poor diet and illness are themselves caused by a set of underlying factors that include family access to food and maternal care-taking practices. Finally, these underlying factors are influenced by the basic socioeconomic and political conditions within which poor families are attempting to raise well-nourished children (Fotso, and KuateDefo, 2005).

An accurate understanding of the relationships among these various causes of malnutrition and the relative contribution of each is essential for the design of

Vol (2) , No (3) , Supplement June 2014 
efficient and effective programs to reduce malnutrition and its consequences. Because the resources directed at improving nutritional status are relatively scarce, it is critical that these resources are directed at interventions that will lead to lasting improvements (The State of the World's Children Geneva, 1998, Dolin et al., 2010).

Dietary intakes unquestionably affect nutritional status and growth. Dietary intakes of infants and young children in developing countries come from a combination of breast milk and complementary (or transitional) foods. The scientific understanding regarding which nutrients are most responsible for the widespread growth faltering seen in developing countries, however, has evolved significantly over the past half-century (Allen,1994),(Brown, et al., 1998). Many dietary practices are affected by culture, both in the types of food eaten and in the approach to progression of infant feeding. Explore the cultural practice of the family related to infant feeding so that you can support the family's cultural values (Ricci and Kyle, 2009).

Newborns and infants are experiencing tremendous growth and need diets that support these rapid changes. The National Association of Pediatric Nurse Practitioners (NAPNAP), the American College of Obstetrics and Gynecology, the American Dietetic Association, and the U.S. Breast feeding committee of the Department of Health and Human Services all recommended breastfeeding as the natural and preferred method of newborn and infant feeding. In their position statement on breastfeeding (2001), NAPNAP identifies "human milk as superior to all substitute feeding methods". Breast milk provides complete infant nutrition (Ricci and Kyle, 2009).

After 6 months of age, infants usually require the nutrients available in solid foods in addition to their breast milk or formula. Progressing to feeding solid food can be exciting and trying. Before solid foods are attempted, the infant should be assessed for readiness to progress. Parents need instruction in choosing appropriate solid foods and support in the progression process (Ricci and Kyle, 2009).

Several factors contribute to the appropriate timing of solid food introduction. Iron fortified rice cereal mixed with a small amount of breast milk is a good choice for the first solid food. Infants and children learn about food within a social context, so the family plays an important role in creating healthy eating habits. Families "model" eating behaviors; infants and children learn about eating through watching others. Lifelong eating patterns are often established in childhood, so it is important to emphasize healthy eating practices beginning in infancy (Ricci and Kyle, 2009).
Knowledge and assessment of growth and development help the nurse provide screening for physical and emotional problems; offer anticipatory guidance to parents and caregivers; develop a rapport with the child to enhance the provision of health care; and provide education to the family to build a healthy lifestyle for the future (Sandra and Nettina , 2010).

All pediatric nurses understand the importance of optimal nutrition for the normal healthy child. The nurse knows that in order for children of all ages to reach the goal of adequate nutrition, up-to-date advice and dietary support must be provided. The body of knowledge in pediatric clinical nutrition has seen great advances over the past few decades (Christina, 2000). Job Duties for the Child Care Nurse Consultant (CCNC): Provides guidance, support, referrals, and access to care coordination for families and child care businesses to access health, nutrition, and safety education and services for children (Iowa Department of Public Health, 2006).

Significance of the study

In developing countries prevalence of malnutrition among the under 5 years is estimated at $27 \%$ (United Nations Children's Fund, 2006). Malnutrition is responsible for $54 \%$ of all deaths among children below 5 years of age. It is important, therefore, that children are properly assessed for evidence of malnutrition (WHO, 2005). Of all children under the age of 5 years in developing countries, about $31 \%$ are underweight, $38 \%$ have stunted growth and $9 \%$ show wasting (Brabin \& Coulter, 2003). Poor knowledge on part of mothers can lead to disastrous results in the field of care giving. If the mothers are not acquainted with knowledge pertaining to feeding, weaning, health and nutrition including growth and behavior of children, it might affect the rearing of their children (Muller et al, 2003), (Kwena, et al., 2003).

Each year about 12 million infants and children die in the developing countries (United Nations, 2002). Specifically in Africa, more than $20 \%$ on average do not reach their fifth birthday. Majority of these deaths are due to infectious and parasitic diseases, while about $54 \%$ of the children die malnourished. In poor countries, children from birth or soon after are caught in a malnutrition-infection cycle, which many do not survive due to poor feeding practices and unhealthy environment (Guandilini, 2004).

\section{Patients and methods Research design}

A descriptive design was utilized for this study. 


\section{Setting}

This study was conducted in all Maternal-Child Health Centers $(\mathrm{MCH})$ at Minia city (first, second, third and fourth $\mathrm{MCH}$ ).

\section{Sample}

A convenient sample of 1000 mothers who have children at the infancy stage in all MCH centers at ElMinia city in 6 months period, which were selected randomly during the period from the first of August 2011 until the end of February 2012.

\section{Tools of the study}

A structured interview sheet was developed by the researcher based on review of relevant literature. It included two parts

Part I: sociodemographic characteristics of the mother and child such as age of child, sex, birth order, birth space, mother's age, level of education, socioeconomic status, level of education and religion.

Part II: Assessment of mothers' knowledge related to breast feeding, bottle feeding, and weaning.

\section{Procedure}

\section{1- Preparatory phase}

An official letter was sent from the dean of the Faculty of Nursing, El-Minia University, to the head of $\mathrm{MCH}$ centers at Minia city asking for permission to collect data. The aim and process of study were briefly explained through direct personal communication with the patients. The researcher developed schedule for performing the research, and participants' interview.

\section{2- Pilot Study}

A pilot study was conducted at the beginning of the study. It included $10 \%$ of the total sample to investigate the feasibility of data collection tools and their clarity. Results of the pilot study did not lead to modifications of questions. Subjects included in the pilot study were excluded from the total studied sample.

\section{3- Data collection}

Data were collected through 6 months in the period from August 2011 to the end of February 2012.The researcher went to the $\mathrm{MCH}$ centers at Minia city, 2 days per week (Saturday, and Wednesday from 9 am to $2 \mathrm{pm}$ because patient follow up done in those 2 days. The number of mothers varied, with an average of 2-3 mothers in each session. The sheet required about 30-45 minutes to fill it.

\section{4- Ethical consideration}

All the relevant principles of ethics in research were followed. The study protocol was approved by the pertinent authority. Participants' consent to participate was obtained after informing them about their rights to participate, refuse, or withdraw at any time. Total confidentiality of any obtained information was ensured. The study maneuver could not entail any harmful effects on participants.

\section{Statistical Analysis}

Data entry and statistical analysis were done using SPSS 11.0 (statistical package for social science), statistical software package. Data were presented using descriptive statistics; comparison between groups of variables was done by Chi-Square test (for non -parametric data). Quantitative data were presented by mean and standard deviation, while qualitative data were presented by frequency distribution. Pearson correlation analysis was used to identify the inter-relationships between variables. Statistical significance was considered at p-value $<0.05$. 


\section{Results}

Table 1. Relation between total knowledge of mothers- about breast feeding and the infant's sociodemographic characteristics No $=(\mathbf{1 0 0 0})$.

\begin{tabular}{|c|c|c|c|c|c|c|c|c|}
\hline & \multicolumn{6}{|c|}{ Total knowledge about breast feeding } & \multirow{3}{*}{$x^{2}$} & \multirow{3}{*}{$P$-value } \\
\hline & \multicolumn{2}{|c|}{ Poor $(\mathrm{No}=711)$} & \multicolumn{2}{|c|}{ Satisfied $(\mathrm{No}=269)$} & \multicolumn{2}{|c|}{ Good $(\mathrm{No}=20)$} & & \\
\hline & No & $\%$ & No & $\%$ & No & $\%$ & & \\
\hline $\begin{array}{l}\text { Age of child } \\
<6 \text { months } \\
\geq 6 \text { months }\end{array}$ & $\begin{array}{l}364 \\
347\end{array}$ & $\begin{array}{l}51.2 \\
48.8\end{array}$ & $\begin{array}{l}132 \\
137\end{array}$ & $\begin{array}{l}49.1 \\
50.9\end{array}$ & $\begin{array}{c}11 \\
9\end{array}$ & $\begin{array}{l}61.1 \\
38.9\end{array}$ & 18.209 & $0.05 *$ \\
\hline $\begin{array}{c}\text { Sex of child } \\
\text { Male } \\
\text { Female } \\
\end{array}$ & $\begin{array}{l}321 \\
390 \\
\end{array}$ & $\begin{array}{l}45.1 \\
54.9 \\
\end{array}$ & $\begin{array}{l}133 \\
136 \\
\end{array}$ & $\begin{array}{l}49.4 \\
50.6 \\
\end{array}$ & $\begin{array}{c}7 \\
13 \\
\end{array}$ & $\begin{array}{l}35.0 \\
65.0 \\
\end{array}$ & 2.461 & $\begin{array}{c}0.292 \\
\mathrm{NS} \\
\end{array}$ \\
\hline $\begin{array}{l}\text { Birth order } \\
1^{\text {st }} \\
2^{\text {nd }}-3^{\text {rd }} \\
4-6^{\text {th }} \\
>6^{\text {th }} \\
\end{array}$ & $\begin{array}{c}260 \\
338 \\
90 \\
23 \\
\end{array}$ & $\begin{array}{c}36.6 \\
47.5 \\
12.7 \\
3.2 \\
\end{array}$ & $\begin{array}{c}139 \\
98 \\
29 \\
3 \\
\end{array}$ & $\begin{array}{c}51.7 \\
36.4 \\
10.8 \\
1.1 \\
\end{array}$ & $\begin{array}{c}19 \\
1 \\
0 \\
0 \\
\end{array}$ & $\begin{array}{c}95.0 \\
5.0 \\
0.0 \\
0.0 \\
\end{array}$ & 48.935 & $0.00001 * *$ \\
\hline $\begin{array}{l}\text { Birth } \text { space } \\
\quad=\text { Prime } \\
=<2 \text { years } \\
2-3 \text { years } \\
=>3 \text { years }\end{array}$ & $\begin{array}{c}261 \\
37 \\
227 \\
186\end{array}$ & $\begin{array}{c}36.7 \\
5.2 \\
31.9 \\
26.2\end{array}$ & $\begin{array}{c}139 \\
17 \\
64 \\
49\end{array}$ & $\begin{array}{c}51.7 \\
6.3 \\
23.8 \\
18.2\end{array}$ & $\begin{array}{c}19 \\
1 \\
0 \\
0\end{array}$ & $\begin{array}{c}95.0 \\
5.0 \\
0.0 \\
0.0\end{array}$ & 45.029 & $0.0001 * *$ \\
\hline
\end{tabular}

$\mathrm{NS}=$ Not significant $\quad *=$ Statistical significant $\quad * *=$ Highly statistical significant

Fig. (1) Relation between total knowledge of mother about breast feeding and age of infant.

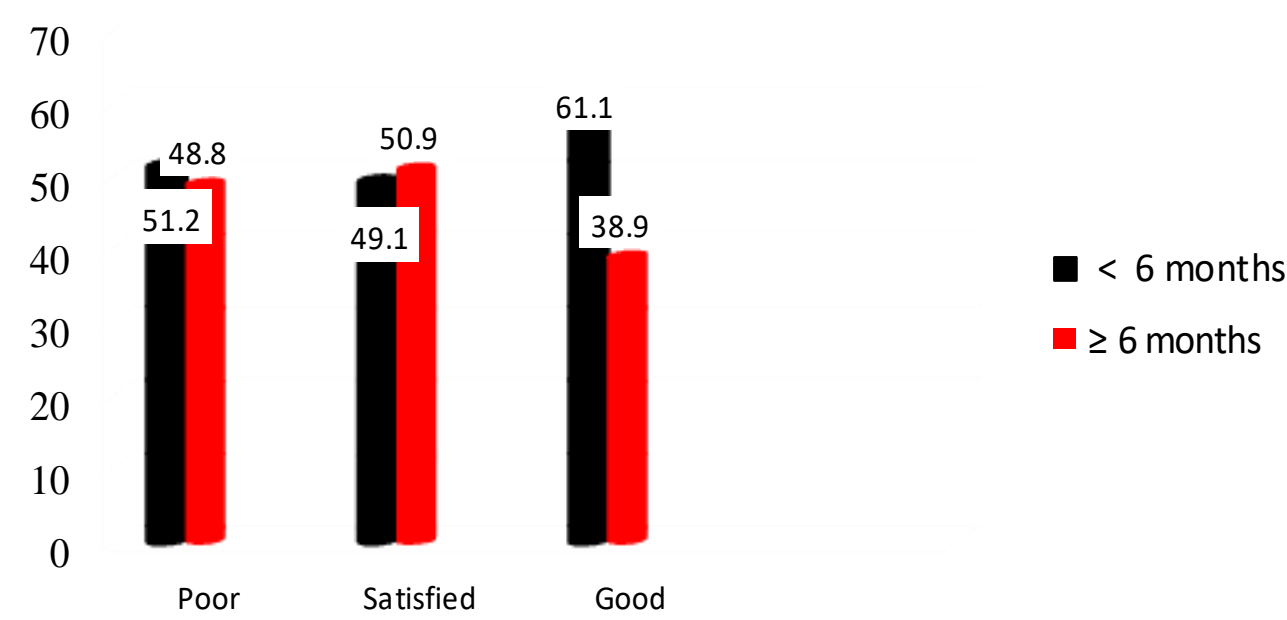


Table 2. Relation between total knowledge of mothers - about breastfeeding and her socio-demographic characteristics. $\mathrm{No}=(\mathbf{1 0 0 0})$.

\begin{tabular}{|c|c|c|c|c|c|c|c|c|}
\hline & \multicolumn{6}{|c|}{ Total knowledge about breast feeding } & \multirow{3}{*}{$\chi^{2}$} & \multirow{3}{*}{$P$-value } \\
\hline & \multicolumn{2}{|c|}{ Poor } & \multicolumn{2}{|c|}{ Satisfied } & \multicolumn{2}{|c|}{ Good } & & \\
\hline & No & $\%$ & No & $\%$ & No & $\%$ & & \\
\hline $\begin{array}{l}\text { Age of the mother } \\
<20 \mathrm{yrs} \\
20-30 \mathrm{yrs} \\
30-35 \mathrm{yrs} \\
>35 \mathrm{yrs} \\
\end{array}$ & $\begin{array}{c}120 \\
476 \\
104 \\
11 \\
\end{array}$ & $\begin{array}{c}16.9 \\
66.9 \\
14.6 \\
1.5\end{array}$ & $\begin{array}{c}13 \\
203 \\
28 \\
25\end{array}$ & $\begin{array}{c}4.8 \\
75.5 \\
10.4 \\
9.3 \\
\end{array}$ & $\begin{array}{c}0 \\
20 \\
0 \\
0 \\
\end{array}$ & $\begin{array}{c}0.0 \\
100.0 \\
0.0 \\
0.0\end{array}$ & 67.265 & $0.0001 * *$ \\
\hline $\begin{array}{l}\text { Socioeconomic status } \\
\text { Low } \\
\text { Moderate } \\
\text { High } \\
\end{array}$ & $\begin{array}{c}356 \\
279 \\
76 \\
\end{array}$ & $\begin{array}{l}50.1 \\
39.2 \\
10.7 \\
\end{array}$ & $\begin{array}{c}2 \\
91 \\
176 \\
\end{array}$ & $\begin{array}{c}0.7 \\
33.8 \\
65.4 \\
\end{array}$ & $\begin{array}{c}0 \\
0 \\
20\end{array}$ & $\begin{array}{c}0.0 \\
0.0 \\
100.0 \\
\end{array}$ & 403.794 & $0.0001 * *$ \\
\hline $\begin{array}{c}\text { Residence } \\
\text { Rural } \\
\text { Urban }\end{array}$ & $\begin{array}{l}469 \\
242\end{array}$ & $\begin{array}{l}66.0 \\
34.0\end{array}$ & $\begin{array}{c}41 \\
228\end{array}$ & $\begin{array}{l}15.2 \\
84.8\end{array}$ & $\begin{array}{c}2 \\
18\end{array}$ & $\begin{array}{l}10.0 \\
90.0\end{array}$ & 214.818 & $0.0001 * *$ \\
\hline $\begin{array}{l}\text { Occupation of mother } \\
\text { Not working } \\
\text { Working }\end{array}$ & $\begin{array}{c}669 \\
42\end{array}$ & $\begin{array}{c}94.1 \\
5.9\end{array}$ & $\begin{array}{c}82 \\
187\end{array}$ & $\begin{array}{l}30.5 \\
69.5\end{array}$ & $\begin{array}{c}0 \\
20\end{array}$ & $\begin{array}{c}0.0 \\
100.0\end{array}$ & 483.833 & $0.0001 * *$ \\
\hline $\begin{array}{l}\text { Education of mother } \\
\text { Illiterate } \\
\text { Read\& write } \\
\text { Basic education } \\
\text { University }\end{array}$ & $\begin{array}{c}390 \\
14 \\
279 \\
28\end{array}$ & $\begin{array}{c}54.9 \\
2.0 \\
39.2 \\
3.9\end{array}$ & $\begin{array}{c}5 \\
0 \\
55 \\
209\end{array}$ & $\begin{array}{c}1.9 \\
0.0 \\
20.4 \\
77.7\end{array}$ & $\begin{array}{c}0 \\
0 \\
2 \\
18\end{array}$ & $\begin{array}{c}0.0 \\
0.0 \\
10.0 \\
90.0\end{array}$ & 661.069 & $0.0001 * *$ \\
\hline
\end{tabular}

Table 3. Relation between total knowledge of mother about bottle feeding and the infant's socio-demographic characteristics, $\mathbf{n o}=(\mathbf{1 0 0 0})$.

\begin{tabular}{|c|c|c|c|c|c|c|c|c|}
\hline & \multicolumn{6}{|c|}{ Total knowledge about artificial feeding } & \multirow{3}{*}{$\chi^{2}$} & \multirow{3}{*}{$P$-value } \\
\hline & \multicolumn{2}{|c|}{ Poor $($ No $=729)$} & \multicolumn{2}{|c|}{ Satisfied(No = 138) } & \multicolumn{2}{|c|}{ Good $($ No $=133)$} & & \\
\hline & No & $\%$ & No & $\%$ & No & $\%$ & & \\
\hline $\begin{array}{l}\text { Age of child } \\
<6 \text { months } \\
\geq 6 \text { months }\end{array}$ & $\begin{array}{l}383 \\
346 \\
\end{array}$ & $\begin{array}{l}52.5 \\
47.5 \\
\end{array}$ & $\begin{array}{l}77 \\
61 \\
\end{array}$ & $\begin{array}{l}55.8 \\
44.2 \\
\end{array}$ & $\begin{array}{l}47 \\
86 \\
\end{array}$ & $\begin{array}{l}35.3 \\
64.7 \\
\end{array}$ & 92.197 & $0.0001 *$ \\
\hline $\begin{array}{l}\text { Sex of child } \\
\text { Male } \\
\text { Female } \\
\end{array}$ & $\begin{array}{l}323 \\
406 \\
\end{array}$ & $\begin{array}{l}44.3 \\
55.7 \\
\end{array}$ & $\begin{array}{l}81 \\
57 \\
\end{array}$ & $\begin{array}{l}58.7 \\
41.3 \\
\end{array}$ & $\begin{array}{l}57 \\
76 \\
\end{array}$ & $\begin{array}{l}42.9 \\
57.1 \\
\end{array}$ & 10.317 & $0.006^{*}$ \\
\hline $\begin{array}{l}\text { Birth order } \\
1^{\text {st }} \\
2^{\text {nd }}-3^{\text {rd }} \\
4-6^{\text {th }} \\
>6^{\text {th }}\end{array}$ & $\begin{array}{c}336 \\
292 \\
81 \\
20 \\
\end{array}$ & $\begin{array}{c}46.1 \\
40.1 \\
11.1 \\
2.7 \\
\end{array}$ & $\begin{array}{c}28 \\
77 \\
27 \\
6 \\
\end{array}$ & $\begin{array}{c}20.3 \\
55.8 \\
19.6 \\
4.3 \\
\end{array}$ & $\begin{array}{c}54 \\
68 \\
11 \\
0 \\
\end{array}$ & $\begin{array}{l}40.6 \\
51.1 \\
8.3 \\
0.0 \\
\end{array}$ & 55.248 & $0.0001 *$ \\
\hline $\begin{array}{l}\text { Birth space } \\
\text { Prime } \\
<2 \text { years } \\
2-3 \text { years } \\
>3 \text { years }\end{array}$ & $\begin{array}{c}337 \\
36 \\
193 \\
163 \\
\end{array}$ & $\begin{array}{c}46.2 \\
4.9 \\
26.5 \\
22.4\end{array}$ & $\begin{array}{c}28 \\
9 \\
55 \\
46 \\
\end{array}$ & $\begin{array}{c}20.3 \\
6.5 \\
39.9 \\
33.3\end{array}$ & $\begin{array}{l}54 \\
10 \\
43 \\
26\end{array}$ & $\begin{array}{c}40.6 \\
7.5 \\
32.3 \\
19.5 \\
\end{array}$ & 35.013 & $0.0001 *$ \\
\hline
\end{tabular}

* = Highly statistical significant 
Table 4. Relation between total knowledge of mother about bottle feeding and her socio-demographic characteristics, $n o=(\mathbf{1 0 0 0})$.

\begin{tabular}{|c|c|c|c|c|c|c|c|c|}
\hline & \multicolumn{6}{|c|}{ Total knowledge about artificial feeding } & \multirow{3}{*}{$x^{2}$} & \multirow{3}{*}{$P$ - value } \\
\hline & \multicolumn{2}{|c|}{ Poor } & \multicolumn{2}{|c|}{ Satisfied } & \multicolumn{2}{|c|}{ Good } & & \\
\hline & No & $\%$ & No & $\%$ & No & $\%$ & & \\
\hline $\begin{array}{l}\text { Age of the mother } \\
<20 \mathrm{yrs} \\
20-30 \mathrm{yrs} \\
30-35 \mathrm{yrs} \\
>35 \mathrm{yrs} \\
\end{array}$ & $\begin{array}{c}126 \\
488 \\
93 \\
22 \\
\end{array}$ & $\begin{array}{c}17.3 \\
66.9 \\
12.8 \\
3.0 \\
\end{array}$ & $\begin{array}{c}7 \\
97 \\
32 \\
2 \\
\end{array}$ & $\begin{array}{c}5.1 \\
70.3 \\
23.2 \\
1.4 \\
\end{array}$ & $\begin{array}{c}0 \\
114 \\
7 \\
12 \\
\end{array}$ & $\begin{array}{c}0.0 \\
85.7 \\
5.3 \\
9.0 \\
\end{array}$ & 69.295 & $0.0001 *$ \\
\hline $\begin{array}{l}\text { Socioeconomic status } \\
\text { Low } \\
\text { Moderate } \\
\text { High }\end{array}$ & $\begin{array}{l}319 \\
284 \\
126\end{array}$ & $\begin{array}{l}43.8 \\
39.0 \\
17.2\end{array}$ & $\begin{array}{l}38 \\
40 \\
60\end{array}$ & $\begin{array}{l}27.5 \\
29.0 \\
43.5\end{array}$ & $\begin{array}{c}1 \\
46 \\
86\end{array}$ & $\begin{array}{c}0.8 \\
34.6 \\
64.7\end{array}$ & 172.943 & $0.00001 *$ \\
\hline $\begin{array}{c}\text { Residence } \\
\text { Rural } \\
\text { Urban }\end{array}$ & $\begin{array}{l}418 \\
311\end{array}$ & $\begin{array}{l}57.3 \\
42.7\end{array}$ & $\begin{array}{l}85 \\
53\end{array}$ & $\begin{array}{l}61.6 \\
38.4\end{array}$ & $\begin{array}{c}9 \\
124\end{array}$ & $\begin{array}{c}6.8 \\
93.2\end{array}$ & 122.056 & $0.00001 *$ \\
\hline $\begin{array}{l}\text { Occupation of mother } \\
\text { Not working } \\
\text { Working } \\
\end{array}$ & $\begin{array}{c}637 \\
92 \\
\end{array}$ & $\begin{array}{l}87.4 \\
12.6 \\
\end{array}$ & $\begin{array}{l}75 \\
63 \\
\end{array}$ & $\begin{array}{l}54.3 \\
45.7 \\
\end{array}$ & $\begin{array}{l}39 \\
94 \\
\end{array}$ & $\begin{array}{l}29.3 \\
70.7 \\
\end{array}$ & 239.608 & $0.00001 *$ \\
\hline $\begin{array}{l}\text { Education of the mother } \\
\text { Illiterate } \\
\text { Read\& write } \\
\text { Basic education } \\
\text { University }\end{array}$ & $\begin{array}{c}360 \\
11 \\
264 \\
94\end{array}$ & $\begin{array}{c}49.4 \\
1.5 \\
36.2 \\
12.9\end{array}$ & $\begin{array}{c}33 \\
3 \\
46 \\
56\end{array}$ & $\begin{array}{c}23.9 \\
2.2 \\
33.3 \\
40.6\end{array}$ & $\begin{array}{c}2 \\
0 \\
26 \\
105\end{array}$ & $\begin{array}{c}1.5 \\
0.0 \\
19.6 \\
78.9\end{array}$ & 465.141 & $0.00001 *$ \\
\hline
\end{tabular}

$*$ = Highly statistical significant

Table 5. Relation between total knowledge of mother about weaning and the infant's socio-demographic characteristics, $n o=(1000)$.

\begin{tabular}{|c|c|c|c|c|c|c|c|c|}
\hline & \multicolumn{6}{|c|}{ Total knowledge about weaning } & \multirow{3}{*}{$\chi^{2}$} & \multirow{3}{*}{$P$ - value } \\
\hline & \multicolumn{2}{|c|}{$\begin{array}{c}\text { Poor } \\
(\mathrm{No}=488) \\
\end{array}$} & \multicolumn{2}{|c|}{$\begin{array}{c}\text { Satisfied } \\
(\mathrm{No}=\mathbf{2 3 0})\end{array}$} & \multicolumn{2}{|c|}{$\begin{array}{c}\text { Good } \\
(\text { No }=282) \\
\end{array}$} & & \\
\hline & No & $\%$ & No & $\%$ & No & $\%$ & & \\
\hline $\begin{array}{l}\text { Age of child } \\
\quad<6 \text { months } \\
\geq 6 \text { months }\end{array}$ & $\begin{array}{l}267 \\
221 \\
\end{array}$ & $\begin{array}{l}65.6 \\
34.4 \\
\end{array}$ & $\begin{array}{l}105 \\
125\end{array}$ & $\begin{array}{l}45.7 \\
54.3 \\
\end{array}$ & $\begin{array}{l}135 \\
147\end{array}$ & $\begin{array}{l}47.9 \\
52.1\end{array}$ & 15.376 & $\begin{array}{c}0.119 \\
\text { NS }\end{array}$ \\
\hline $\begin{array}{l}\text { Sex of child } \\
\text { Male } \\
\text { Female }\end{array}$ & $\begin{array}{l}226 \\
262\end{array}$ & $\begin{array}{l}46.3 \\
53.7\end{array}$ & $\begin{array}{l}101 \\
129\end{array}$ & $\begin{array}{l}43.9 \\
56.1\end{array}$ & $\begin{array}{l}134 \\
148\end{array}$ & $\begin{array}{l}47.5 \\
52.5\end{array}$ & 0.680 & $\begin{array}{c}0.712 \\
\text { NS }\end{array}$ \\
\hline $\begin{array}{l}\text { Birth order } \\
1^{\text {st }} \\
2^{\text {nd }}-3^{\text {rd }} \\
4-6^{\text {th }} \\
>6^{\text {th }}\end{array}$ & $\begin{array}{c}203 \\
202 \\
67 \\
16\end{array}$ & $\begin{array}{c}41.6 \\
41.4 \\
13.7 \\
3.3\end{array}$ & $\begin{array}{c}78 \\
126 \\
23 \\
3\end{array}$ & $\begin{array}{c}33.9 \\
54.8 \\
10.0 \\
1.3\end{array}$ & $\begin{array}{c}137 \\
109 \\
29 \\
7\end{array}$ & $\begin{array}{c}48.6 \\
38.6 \\
10.3 \\
2.5\end{array}$ & 23.295 & $0.003 *$ \\
\hline $\begin{array}{c}\text { Birth space } \\
<2 \text { years } \\
2 \text { years } \\
3 \text { years } \\
>3 \text { years }\end{array}$ & $\begin{array}{c}203 \\
32 \\
153 \\
100\end{array}$ & $\begin{array}{c}41.6 \\
6.6 \\
31.4 \\
20.5\end{array}$ & $\begin{array}{c}79 \\
7 \\
61 \\
83\end{array}$ & $\begin{array}{c}34.3 \\
3.0 \\
26.5 \\
36.1\end{array}$ & $\begin{array}{c}137 \\
16 \\
77 \\
52\end{array}$ & $\begin{array}{c}48.6 \\
5.7 \\
27.3 \\
18.4\end{array}$ & 31.824 & $0.00001 *$ \\
\hline
\end{tabular}


Table 6. Relation between total knowledge of mother about weaning and her socio-demographic characteristics, $n o=(\mathbf{1 0 0 0})$.

\begin{tabular}{|c|c|c|c|c|c|c|c|c|}
\hline & \multicolumn{6}{|c|}{ Total knowledge about weaning } & \multirow{3}{*}{$\chi^{2}$} & \multirow{3}{*}{$P$ - value } \\
\hline & \multicolumn{2}{|c|}{ Poor $(\mathrm{No}=488)$} & \multicolumn{2}{|c|}{ Satisfied(No= 230) } & \multicolumn{2}{|c|}{ Good $(\mathrm{No}=282)$} & & \\
\hline & No & $\%$ & No & $\%$ & No & $\%$ & & \\
\hline \multicolumn{9}{|l|}{ Age of the mother } \\
\hline$<20 \mathrm{yrs}$ & 93 & 19.1 & 35 & 15.2 & 5 & 1.8 & \multirow{4}{*}{75.752} & \multirow{4}{*}{$0.00001 *$} \\
\hline $20-30 \mathrm{yrs}$ & 310 & 63.5 & 166 & 72.2 & 223 & 79.1 & & \\
\hline $30-35$ yrs & 79 & 16.2 & 21 & 9.1 & 32 & 11.3 & & \\
\hline$>35 \mathrm{yrs}$ & 6 & 1.2 & 8 & 3.5 & 22 & 7.8 & & \\
\hline \multicolumn{9}{|l|}{ Socioeconomic status } \\
\hline Low & 284 & 58.2 & 73 & 31.7 & 1 & 0.4 & \multirow{3}{*}{450.730} & \multirow{3}{*}{$0.00001^{*}$} \\
\hline Moderate & 182 & 37.3 & 102 & 44.3 & 86 & 30.5 & & \\
\hline High & 22 & 4.5 & 55 & 23.9 & 195 & 69.1 & & \\
\hline \multicolumn{9}{|l|}{ Residence } \\
\hline Rural & 339 & 69.5 & 128 & 55.7 & 45 & 16 & \multirow[b]{2}{*}{207.181} & \multirow[b]{2}{*}{$0.00001^{*}$} \\
\hline Urban & 149 & 30.5 & 102 & 44.3 & 237 & 84 & & \\
\hline \multicolumn{9}{|l|}{ Occupation of mother } \\
\hline Not working & 468 & 95.9 & 218 & 94.8 & 65 & 23 & \multirow{2}{*}{569.132} & \multirow[b]{2}{*}{$0.00001 *$} \\
\hline Working & 20 & 4.1 & 12 & 5.2 & 217 & 77 & & \\
\hline \multicolumn{9}{|l|}{ Education of the mother } \\
\hline Illiterate & 314 & 64.3 & 80 & 34.8 & 1 & 0.4 & \multirow{4}{*}{685.367} & \multirow{4}{*}{$0.00001^{*}$} \\
\hline Read\& write & 6 & 1.2 & 8 & 3.5 & 0 & 0.0 & & \\
\hline Basic education & 158 & 32.42 .1 & 114 & 49.6 & 64 & 22.6 & & \\
\hline University & 10 & & 28 & 12.1 & 217 & 77.0 & & \\
\hline
\end{tabular}

Table (1) : Points to the relation between total knowledge of mother about breast feeding and the infant's socio-demographic characteristics. It shows that there were highly statistical significant difference between total knowledge of mother about breast feeding and all socio-demographic characteristics except the age and sex of infant where $p$ - value $=$ $(0.052,0.292)$ respectively. As well as good knowledge was higher among: age group < 6 months, female child group, $1^{\text {st }}$ birth order and prime birth space.

Figure (1) : Shows the relation between total knowledge of mother about breast feeding age of infant. It indicates that the mothers $(61.1 \%)$ who have good knowledge about breast feeding were having children aging less than six months, while the majority of mothers $(50.9 \%)$ who have fair knowledge about breast feeding were having children aging equals or more than six months.

Table (2) : presents the relation between total knowledge of mothers - about breast feeding and her socio-demographic characteristics. It indicates highly statistical significant difference between total knowledge of mother about breast feeding and all socio-demographic characteristics. As well as good knowledge was higher among: age group 20- 30 years high socioeconomic status, urban area, working mothers, and university mother education.

Table (3) presents the relation between total knowledge of mother about bottle feeding and the infant's socio-demographic characteristics. It points to highly statistical significant difference between total knowledge of mother about bottle feeding and all socio-demographic characteristics. As well as good knowledge was higher among: age group $\geq 6$ months, female child group, $2^{\text {nd }}-3^{\text {rd }}$ birth order and prime birth space.

Table (4) : Presents the relation between total knowledge of mother about bottle feeding and her socio-demographic characteristics - attending $\mathrm{MCH}$ centers at Minia city. There were highly statistical significant difference between total knowledge of mother about bottle feeding and all sociodemographic characteristics. As well as good knowledge was higher among: age group 20- 30 years, high socioeconomic status, urban area, working mothers, and university mother education.

Table (5) : Shows the relation between total knowledge of mother about weaning and the infant's socio-demographic characteristics. There were highly statistical significant difference between total knowledge of mother about weaning and all sociodemographic characteristics except the age and sex of child where $p$ - value $=(0.119,0.712)$ respectively. As well as good knowledge was higher among: age group $\geq 6$ months, female child group, $1^{\text {st }}$ birth order and $<2$ years birth space.

Table (6) : Presents the relation between total knowledge of mother about weaning and her sociodemographic characteristics - attending $\mathrm{MCH}$ centers at Minia city. There were highly statistical significant difference between total knowledge of mother about bottle feeding and all socio-demographic characteristics. As well as good knowledge was 
higher among: age group 20- 30 years, high socioeconomic status, urban area, working mothers, and university mother education.

\section{Discussion}

Our study revealed that higher infant feeding scores correlated with higher maternal age. The results have indicated that the age of the mothers had its effect on their level of knowledge was better among older mothers over 20 years. This difference was statistically significant. These results agreed with Zhou et al., (2010) study on knowledge and attitudes towards breastfeeding in Ireland who stated that those having inaccurate breastfeeding knowledge were more likely to be younger (30 years old), less educated and less affluent mothers. Similar finding has been reported in many other studies like Narayan, (2005) and Khattak' et al., (2007). Better breastfeeding scores also correlated with maternal occupation with professionals having a better knowledge than unskilled workers/housewives .We also found a positive association between breastfeeding and maternal education status similar to a few other studies Wen, et al., (2009). Sheikh et al., (2010) found no definite association of socioeconomic condition of families with their knowledge about feeding of their infants was observed except maternal education.

Based on the results it was observed that there were highly statistical significant difference between total knowledge of mother about breast feeding and all her socio-demographic characteristics. These findings are in accordance with Scott et al., (2009) who found that midwives with personal breastfeeding experience had higher attitude scores than those without. Also Lande et al., (2003) and Al-sahab et al., (2008) found that high parity was also positively associated with 6-month exclusive breastfeeding. A dose response relationship between parity and breastfeeding has been previously documented in the literature.

Multipara mothers are suggested to have increased knowledge and self confidence from earlier breastfeeding experiences. By the same token, young age at first pregnancy decreased the likelihood of 6-month exclusive breastfeeding. Dubois et al., (2003), Lande et al., (2003). Khattak, et al., (2007) noticed that associations with delayed weaning were noted with parity of the mother, large family size and bottle-feeding. Delayed weaning was particularly noticeable when the mother had 5 or more children. This could be due to poor time management of the mother, as they tend to neglect the nutritional needs of the young child. These results disagreed with that conducted by Sheikh et al., (2010) who found no definite association of socioeconomic condition of families with their knowledge about feeding of their infants was observed except maternal education.

Our results revealed that there was a positive relationship between maternal education and increased knowledge about infant feeding. These results are in accordance with the study conducted in Islamabad Pakistan by Galhotra et al., (2008), which found a positive relationship between the nutritional status of infants and maternal education. The study revealed that majority of malnourished infants belonged to mothers with virtually no school education.

This was supported by the research conducted in Lahore survey in an urban community of Lahore, (2005). Maternal education was the only predictor for use of pre-lacteal fluids, with uneducated mothers more likely to give pre-lacteals than their educated counterparts. The reasons for giving pre-lacteals documented in various studies were 'waiting for spontaneous milk flow and purging the gut of meconium, there is no literature to explain why mothers indulge in this practice. This suggests that despite improvement in breastfeeding practices there is little change due to strong cultural beliefs Memon et al., (2008).

According to the mothers' occupation, our study revealed that working mothers have better knowledge about exclusive breast feeding than non-working mothers. These results are in accordance with Scott et al., (2009)who found that the prevalence of exclusive breastfeeding was better among working mothers than nonworking mothers.

These results disagreed with the study conducted by Lakshman et al., (2011) showed that factors associated with not practicing full breastfeeding were mothers' working status and delivery by caesarean section, where mothers rarely care for their babies in the first 2 days post-operatively.

In the same line, Mohamed et al., (2006) found that employed women were more likely not to practice full $\mathrm{BF}$ compared to unemployed women and women who had caesarian delivery were more likely not to practice full breastfeeding compared to those who had vaginal delivery.

Also, Fayed, et al., (2012), EI- Wahab and Zekry, (2009) found that $(93 \%)$ of non working mothers breastfeed their infants on cue, compared to only $(8 \%)$ of working mothers. This can be explained by work condition that interferes with cue feeding. Risk, et al., (2003) found that $(67.7 \%)$ of non working and $(40 \%)$ of working group feed their infants on cue.

Leong (2009) found that working women were more likely not to practice exclusive breastfeeding compared to non working women in Malaysia. Arts 
and his colleagues found that only less than half of infants younger than 6 months in Mozambique were exclusively breastfed. The practice of exclusive breastfeeding depends on various factors related to both mothers and their environment. Exclusive breastfeeding is not promoted in healthcare facilities because the health professionals do not encourage it. Moussa Abba et al., (2010).

Fayed, et al., (2012) found that there was statistically significant difference as regard exclusive breast feeding duration. The results showed that: The exclusive breastfeeding to six months was significantly higher among non working mothers versus in working mothers. The mean age of onset of complementary feeding also show statistically significant difference the mean duration was (6.1) months in non working mothers versus (4.5) months in working mothers.

Millar and Maclean (2005) revealed that postsecondary education was positively associated with exclusive breastfeeding for the first 6 months of life. Similarly in Quebec, having a university diploma increased the odds of 4-month exclusive breastfeeding Dubois et al., (2003) and not completing high school was a risk factor for early breastfeeding termination in Ontario. A higher level of maternal education seems to allow mothers to formulate well-informed decisions regarding the feeding practices used for their infant.

Chema et al., (2011) Analysis also revealed that mothers accepted bottle feeding due to certain factors - which are type of work, mother's ill health, and insufficient milk production, respectively. This corroborates the work of Cukier (1999) and Arora and Wehrer (2000) that bottle feeding was started due to insufficient milk, work outside home, lack of desire to breastfeed, mother's ill health and uncertainty regarding quantity of milk. According to Schiess et al., (2010) only a slight minority of mothers considered formula or dairy milk more beneficial as compared to breast milk whereas a large majority of mothers and all doctors and paramedics agreed on breast milk being most beneficial.

Fayed, et al.,(2012) found that the majority of working mothers start weaning after returning to work while nearly half of non working group start weaning due to insufficient breast milk. These results were in agreement with Risk et al., (2003). Khattak, et al, (2007) study on evaluation of nutritional knowledge of mothers about their children in Pakistan stated that early weaning was noted in only (12/105) infants. (41/105) infants were weaned in 4-6 months and delayed weaning was noted in $(52 / 105)$ infants Delayed weaning was noted in $49.5 \%$ cases.

Returning to work was also a strong predictor of early weaning in this sample. Census and Statistics Department,(2008). Work has been frequently cited as a reason for early weaning from breastfeeding. Breastfeeding mothers in Hong Kong have reported that breastfeeding after returning to work in not feasible for them, and thus with their long work days, they must wean before returning to work (Tarrant et al., 2010).

Our study revealed that the majority of the studied mothers stated that clostrum is beneficial. These results are in accordance with Schmidl et al., (2009) as supported by others (first 6 months and associated factors in a rural and semi urban community in Abdul Ameer et al., (2008) concluded that giving clostrum was favored by nearly all of the mothers.

In comparison to this, another study in Pakistan by Memon et al., (2008) showed that $71 \%$ of (rural and urban) mothers discarded colostrums. This shows the importance of early education to mothers who deliver at hospitals, regarding optimum breastfeeding by the hospital staff.

\section{Conclusions}

Based on the results of the present study, we concluded that there is a relationship between the knowledge of mother about breast feeding, bottle feeding, and weaning and her socio-demographic characteristics and that there is a relationship between the education of mother with her knowledge about pattern of breast feeding, knowledge about exclusive breast feeding, duration of exclusive breast feeding, bottle feeding, and weaning and that there is a relationship between the occupation of mother and pattern of breast feeding, knowledge about exclusive breast feeding, duration of exclusive breast feeding.

\section{Recommendations}

Based on the previous findings of the present study, the following recommendations are suggested:

- All the family members, particularly the elderly females should be taught about the disadvantages of prelacteal feeding and importance of colostrums' feeding.

- Mothers should be advised to initiate breast feeding within one hour of delivery.

- Importance of exclusive breast feeding for the first 6 months of baby's life and proper weaning thereafter should be properly explained to the mother.

- The risks of infant formula supplementation, both to the baby's health and to the process of breastfeeding, need to be reinforced to all mothers.

- The ten steps of successful breast feeding and other Infant and child welfare programmes should be followed and practiced by health personnel. 
- Mothers attending the Immunization Clinic, antenatal and postnatal wards should be informed about benefits of breast feeding and different aspects of infant feeding.

\section{References}

1. Abd-El-Wahab M., and Zekry A., (2009): Effects of Maternal Employment on Breast feeding practice in suze Governorate M.Sc Thesis-pediatric, Faculty of medicine, Suez Canal University, Egypt. P465.

2. Abdul Ameer A., AI-Hadi A., and Abdulla M., (2008): Knowledge, attitudes and practices of Iraqi mothers and family child-caring women regarding breastfeeding. East Mediterr Health J 2008; 14: 1003-14.

3. Ali S., Ali S., Imam. A., Ayub S., and Billoo A., (2011): Perception and practices of breastfeeding of infants 0-6 months in an urban and a semi-urban community in Pakistan: a cross-sectional study, JPMA 61:99, 61, (1), pp.99-104.

4. Allen L., (1994): Nutritional influences on linear growth: a general review; 48: 75-89.

5. Al-Sahab B., l.anes' A., Feldman M., and Tarnlm H., (2010): Prevalence and predictors of 6-month exclusive breastfeeding among Canadian women: a national survey, 'Kinesiology \& Health Science, York University, Toronto, Ontario, Canada, 01. BMC Pediatrics, 10:20 http://www.biomedcentral.com/ 14712431/10/20

6. Arora S., Junkin C., Wehrer J., and Kuhn P., (2000): Major Factors Influencing Breastfeeding Rates: Mother's Perception of Father's Attitude and Milk Supply, PEDIATRICS, 106, (5), I06; e67

http://pediatrics.aappublications.org/contentl106/ S/e67.full.html

7. Brabin B., and Coulter J., (2003): Nutritionassociated disease, Manson's tropical diseases. London: Saunders; pp. 561-80.

8. Brown K., Dewey K., and Allen L., (1998): Complementary Feeding of Young Children in Developing Countries: A Review of Current Scientific Knowledge. Geneva: WHO.pp.83:678.

9. Census and Statistics Department, (2008): Hong Kong annual digest of statistics: 2008 edition. Census and Statistics Department. Hong Kong 2008.

10. Chema G., and Chigbo R., (2011): Perception about Bottle Feeding among Mothers in selected health care settings in Aba South Iga, Abia state, Nigeria, Grimard School of Midwifery Anyigba, Kogi State - Nigeria, Continental J. Nursing Science 3 (1): 1 - 6, 2011, Wilolud Journals, $\begin{array}{llll}\text { ISSN: } & 2141 & - & 4173\end{array}$ http://www.wiloludjournal.com

11. Christina J., (2000): Infant Nutrition in the First Year of Life: Tradition or Science.

12. Cukier R., (1999): Reasons for weaning and the introduction of bottle feeding: an alternative approach. Journal of Public Health, 22 (3) 184191. Saude Publication

13. Di Napoli A., Di Lallo D., Pezzotti P., Forastiere F., and Porta D., (2006): Effects of parental smoking and level of education on initiation and duration of breastfeeding. Acta Paediatr, 95:678-685.

14. Dolin, Gerald L., Mandell, John E., Bennett, and Raphael (2010): Mandell, Douglas, and Bennett's principles and practice of infectious diseases (7th ed.). Philadelphia, PA: Churchill Livingstone/Elsevier. pp. Chp 93. ISBN 0-44306839-9. Malnutrition at eMedicine

15. Dubois L., and Girard M., (2003): Social determinants of initiation, duration and exclusivity of breastfeeding at the population level: The results of the longitudinal study of child development in Quebec (ELDEQ 19982002). Can J Public Health 2003, 94:300-305.

16. Duijts L., Jaddoe V., Hofman A., and Moll H. (2010): Prolonged and exclusive breastfeeding reduces the risk of infectious diseases in infancy. Pediatrics.126: e18-e25.

17. Ebrahim B., AI-Enezi A., AI-Turki M., AITurki A., AI-Rabah F, Hammoud M., and AITaiar A., (2011): Knowledge, Misconceptions, and Future Intentions Towards Breastfeeding Among Female University Students in Kuwait, Journal of Human Lactation 27(4) 358-366, http://jhl.sagepub.com/contentl27/4/358

18. Egypt Demographic and Health Survey (EDHS) (2008): Infant feeding and maternal and child nutrition, National Population Council, Macro International Inc. Calverton. Cairo, Egypt, 30-32,157-159,199-202,149- 153.

19. Ekambaram M., Bhat V., and Ahamed M., (2010): Knowledge, attitiude and practice of breastfeeding among postnatal mothers, Jawaharlal Institute of Postgraduate Medical Education and Research, Puducherry, India ,Curr Pediatr Res, 14 (2): 119-124

20. Ergenekon-Ozelci P., Elmaci N., Ertem M., and Saka G, (2006): Breastfeeding beliefs and practices among migrant mothers in slums of Diyarbakir, Turkey, 2001. fur J Public Health, 16(2):143-148

21. Fayed S., Almorsy E., Fathi N., and Wahbyl, (2012): The Effect of Maternal Employment on Breast Feeding Practice among Egyptian Children , Pediatric Department.' Community 
and Occupational medicine department. Faculty of medicine for girls Al-Azhar University, Cairo, Egypt. e.almorsy@gmail.com, Journal of American Science, 8(2):523- 528. http://www.americanscience.org

22. Food and Nutrition Board, (2003): National Academy of Sciences-National Research Council (www.cfsan.fda.gov)

23. Fotso, J., and Kuate-Defo, B., (2005): "Measuring socioeconomic status in health research in developing countries: Should we be focusing on households, communities or both?" Social Indicators Research 72 (2): 189. Doi: 10.1007/s11205-004-5579-8.

24. Galhotra, Abrol A., Agarwal N., Goel N., and Swami H., (2008): Impact of community based awareness campaign on breast- feeding among lactating women in Chandigarh. Internet J Health 2008; 7.

25. Guandalini S., (2004): Textbook of pediatric gastroenterology and nutrition, 1st ed., Taylor \& Francis Group, London

26. Iowa Department of Public Health, (2006): Issue Brief: Status of the Nurse Workforce in Iowa.

27. Kamla R., (2006): Impact of educational intervention on knowledge of mothers regarding childcare and nutrition in Himachal Pradesh, India, pp.139- 42.

28. Kar B., Rao S., and Chandramouli, (2008): Cognitive development in children with chronic protein energy malnutrition, Behav Brain Funct. 2008;

$4: 31$.

doi: $\quad 10.1186 / 1744-9081-4-31$ PMCID: PMC2519065

29. Khattak A., GuF Sh, Muntaha S., ,and Jamaluddln, (2007): Evaluation of Nutritional knowledge of Mothers about their Children, 'Department of Pathology and 2Medicine, Gomal Medical College, D.I.Khan, Pakistan, Gomal Journal of Medical Sciences Jan-June, 2007, 5 (1) $; 17-21$.

30. Kotb S., Mohamed A 1., Mohamed E 2., and Abdel Khalek E 3., (2012): Knowledge and Practices of Working Mother about Breastfeeding and Weaning In Assiut City, Egypt, Life Science Journal, 9(1) 80380http://www.lifesciencesite.com

31. Kruger R., and Gericke G., (2004): A., qualitative approach for exploration of feeding practices, knowledge, and attitudes on child nutrition framework. Tydskrifvir Gesinsekologieen Verbruikerswetenskappe; 32. ISSN 0378-5254.

32. Kruger R., and Gericke G., (2001): Breastfeeding practices of mothers with children (aged 0-36 months) in a rural area of South Africa. A qualitative approach. Journal of Family Ecology and Consumer Sciences; 29: 60-71.

33. Kwena A., Terlouw D., de Vlas S., PhillipsHoward P., Hawley W., and Friedman J., (2003): Prevalence and severity of malnutrition in pre-school children in a rural area of western Kenya ; 68:94-9.

34. Kyle T., (2008): Essentials of pediatric nursing, Growth and development of newborn and infant, $1^{\text {st }}$ ed, Lippincott Williams\& Wilkins comp, pp.71:105.

35. Lakshman R., Landsbaugh J., Schiff A., Hardernarr' W., Kong K, and Griffin S., (2011): Development of a questionnaire to assess maternal attitudes towards infant growth and milk feeding practices. International Journal of Behavioral Nutrition and Physical Activity, 8:35 http://www .ijbn pa.org/content/8/1 /35

36. Lande B., Andersen L., Baerug A., rygg K., Lund-Larsen $K$, veiercd $M B$, and Bjorneboe G., (2003): Infant feeding practices and associated factors in the first six months of life: The Norwegian infant nutrition survey. Acta Paediatr 2003, 92152-161

37. Lanting C., Van Wouwe J., and Reijneveld S., (2005): Infant milk feeding practices in the Netherlands and associated factors. Acta Poediatr, 94:935-942.

38. Leong. L., (2009): Knowledge, attitude and practice on breastfeeding in Klang, Malaysia. The International Medical Journal; 8 (1): 17-22.

39. Levitsky D., and Strupp B., (1995): Malnutrition and the Brain: changing concepts, changing concerns. J Nutr; 125.

40. Liaqat P., Rizvi MA, Qayyum A., and Ahmed H., (2007): Association between complementary feeding practice and mothers' education status in Islaruabad. J Huruan Nutr Dietetics, 20: 340-4.

41. Martorell R., (1997): Under nutrition during pregnancy and early childhood: consequences for cognitive and behavioral development. In: Early Child Development: Investing in Our Children's Future. Young ME, Amsterdam, Elsevier Science.

42. Memon S., Shaikh S., Kousar T., Memon Y., and Rubina, (2010): Assessment of infant feeding practices at a tertiary care hospital, Department of Paediatric, LUMHS Jamshoro, Hyderabad. J Pak Med Assoc, J Pak Med Assoc, 60 (12): 1010-1015.

43. Millar W., and Maclean H., (2005): Breastfeeding practices. Health reports, 16:23-31

44. Moffat T., (2002): Breast feeding wage labor, and insufficient milk in peri-urban Kathmandu. 
Canada Med Anthropol, Apr- Jun; 21 (2); $207-$ 230.

45. Mohammad H., Qadri , Ridah A., AI- Harfi, Mohammad A., and Al-Gamdi, (2006): CDD Program, Diarrhea Control Center, Dammam, Saudi Arabia,.

46. Moussa Abba A., De Koninck M., and Hamelin A., (2010): A qualitative study of the promotion of exclusive breastfeeding by health professionals in Niamey, Niger International Breastfeeding Journal; 5:8.

47. Müller O., Garenne M., Kouyaté B., and Becher H., (2003): The association between protein- energy malnutrition, malaria morbidity and all-cause mortality in West African children., 8:507.

48. Nair K., and Radhakrishnan R., (2004): Early Childhood Development in Deprived Urban Settlements. From Child Development Centre, Medical College, India and *Mahatma Gandhi University, Kerala. Child Development Centre, Medical College, Indian Pediatrics 41:227-237

49. Narayan S., Natarajan N., and Bawa K., (2005): Maternal and Neonatal Factors Adversely Affecting Breastfeeding in the Perinatal Period. Med J Armed Forces Ind 2005; 61: 216-219.

50. Ricci S., \& Kyle T., (2009): Maternity and Pediatric Nursing, Growth and Development of the Newborn and Infant, Lippincott Williams \&Wilkins comp., China, pp 769-798

51. Risk, S., Ragab, A., and EL-Guindy S., (2003): Breastfeeding practices among working and non working mothers and their infants' growth and development, MD Thesis, Faculty of Nursing, Cairo University, Egypt p. 154-216.

52. Sandra M., and Nettina, (2010): Lippincott Manual of Nursing Practice, 9th ed, Lippincott Williams \& Wilkins Philadelphia, chapter 40,

53. Sheeba SS, Nair MKC, Radhakrishnan, RS, Padmamohan J., and Letha S., (2003): Measurement of home environment of preschool children using HOME Inventory, Thesis submitted for Diploma in Clinical child Development, Directorate of Medical Education, Kerala.

54. Schiess S., Grote V., Scaglioni S., Luque V., Martin F., Stolarczyk A., Vecchi F.,and Koletzko B., (2010): Introduction of complementary feeding in 5 European countries. J. Pediatr. Gastroenterol. Nutr. 50:92-98. doi: 10.1097/MPG.0b013e31819f1ddc.

55. Schmidl, M., and Labuza, T., (2009): Infant formula and Medical Foods. In Essential of Functional Foods". Aspen Publishers. pp. 137164. Google Book Search.
56. Scott J., Binns C., Graham K., and Oddy W., (2009): Predictors of the early introduction of solid foods in infants: Results of a cohort study. BMC Pediatr. 9:60. doi: 10.1186/1471-2431-960.

57. Tarrant, M., Fong, D., WuIrene, K., Lee, E., Alice Sham, W., Lam, C., and Dodgson, J., (2010): Breastfeeding and weaning practices among Hong Kong mothers: A prospective study. Tarrant et 01.BMC Pregnancy Childbirth, 10: 27. hUp:llwww.biomedcentral.com/14712393/10/27

58. Tarrant R., Younger K., Sheridan-Pereira M., White M., and Kearney J., (2010): Factors associated with weaning practices in term infants: A prospective observational study in Ireland. Br. J. Nutr. 104:1544-1554. doi: 10.1017/S0007114510002412.

59. Tengku T., and Sulaiman Z., (2010): Reliability and validity of a Malay-version questionnaire assessing knowledge of breastfeeding, Malaysian J Med Sci. Jul-Sep. 17(3): 32-39 www.usm.my/mjms

60. United Nations Children's Fund, (2006): Malnutrition -the challenge. Available online at: URL

:http://www.childonfo.org/areas/malnutrition /index.php, Missed opportunities for diagnosis of malnutrition.p.75.

61. United Nations.(2002): Facts for Life. Third Ed. 2002: 75.

62. Wen L., Baur L., Rissel C., Alperstein G., and Simpson M., (2009): Intention to breastfeed and awareness of health recommendations: findings from first-time mothers in southwest Sydney, Australia. Int Breastfeed J, 4:9. doi:10.1186/1746-4358-4-9.

http://www.internationalbreastfeedingjournal.co $\mathrm{m} /$ content14/ I 19 C

63. World Health Organization, (2005): Handbook IMCI Integrated Management of Childhood Illness.

64. World Health Organization (2008): Indicators for assessing infant and young child feeding practices, part 1-definitions: conclusions of a consensus meeting held 6-8 November 2007 in Washington D.C., USA. Geneva, WHO.

65. Zhou Q., Younger K., and Kearney J., (2010): An exploration of the knowledge and attitudes towards breastfeeding among a sample of Chinese mothers in Ireland, 01. BMC Public Health, $\quad 10: 722$ http://www.biomedcentral.com/147124S8/10/722(http://www.who.int/foodsafety/pub lications/ micro/en/es.pdf). 